\title{
On cubic Thue inequalities and a result of Mahler
}

\author{
by \\ JefFrey Lin Thunder (DeKalb, Ill.)
}

Introduction. Let $F(X, Y) \in \mathbb{Z}[X, Y]$ be a form of degree $d \geq 3$ with integral coefficients which is irreducible over the rational numbers $\mathbb{Q}$. It is well known that the number of integral solutions $(x, y) \in \mathbb{Z}^{2}$ to the Thue inequality $|F(x, y)| \leq m$ is finite. Moreover, one can estimate the number $N_{F}(m)$ of such solutions.

Let $A(F)$ denote the area of the region $\left\{(x, y) \in \mathbb{R}^{2}:|F(x, y)| \leq 1\right\}$. Mahler in [M] approximates $N_{F}(m)$ by $m^{2 / d} A(F)$, getting

$$
\left|N_{F}(m)-m^{2 / d} A(F)\right|=O\left(m^{1 /(d-1)}\right)
$$

as $m \rightarrow \infty$, where the constant implicit in the $O$ notation depends on $F$. More recently, W. Schmidt showed that $N_{F}(m) \ll m^{2 / d}(d+\log m)$, where the implied constant is absolute ([S], Chap. 3, Th. 1C), and later the author in [T2] proved essentially that $N_{F}(m) \ll d m^{2 / d}$. See also [MS1], [MS2].

Now on the one hand, Mahler's result (1) is better in that $m^{2 / d} A(F)$ really should approximate $N_{F}(m)$. But on the other hand, $N_{F}(m)$ should be bounded above by some function of $m$ and $d$ independent of the coefficients of $F$, as it is in the latter two results mentioned above. While it is known that $A(F)$ is bounded above (see [B2]), the implicit constant in the error term of (1) actually grows polynomially with the height of $F$ (see [B1]). The obvious conjecture is that $N_{F}(m)$ and $m^{2 / d} A(F)$ should differ by a function depending only on $m$ and $d$.

The author in [T1] proved just such a result in the case of cubic forms, i.e., when the degree $d=3$ :

$$
\left|N_{F}(m)-m^{2 / 3} A(F)\right|=O\left(1+m^{29 / 44} \log m\right)
$$

as $m \rightarrow \infty$, where the constant implicit in the $O$ notation is absolute. Besides the fact that the error term here, as a function of $m$, is larger than that in (1), this result is lacking in another way which we now describe.

1991 Mathematics Subject Classification: 11D75, 11J25.

Research partially supported by NSA grant MDA904-95-1-1087. 
Suppose $G(X, Y) \in \mathbb{R}[X, Y]$ is a form of degree $d \geq 3$ with discriminant $D(G) \neq 0$. For $T \in \mathrm{GL}_{2}(\mathbb{R})$ we get a form $G^{T}(X, Y)=G(T(X, Y))$. Two important observations here are first that the product $A(G)|D(G)|^{1 /(d(d-1))}$ is invariant under such actions, i.e.,

$$
A\left(G^{T}\right)\left|D\left(G^{T}\right)\right|^{1 /(d(d-1))}=A(G)|D(G)|^{1 /(d(d-1))}
$$

(see [B2]), and second that $N_{F}(m)$ is invariant under the action by $T \in$ $\mathrm{GL}_{2}(\mathbb{Z})$.

Now suppose $F(X, Y) \in \mathbb{Z}[X, Y]$ is a cubic form with a non-zero discriminant. Such a form is equivalent under the action of $\mathrm{GL}_{2}(\mathbb{R})$ to either $X Y(X-Y)$ or $X\left(X^{2}+Y^{2}\right)$, depending on whether it factors over $\mathbb{R}$ or not. In particular, there are only two possible values for the product $A(F)|D(F)|^{1 / 6}$ (namely $3 B(1 / 3,1 / 3$ ) and $\sqrt{3} B(1 / 3,1 / 3$ ), where $B$ is the standard beta function). Thus, for such forms $F$, the main term $A(F) m^{2 / 3}$ in (1) and (2) above essentially decreases as a function of the absolute value of the discriminant $|D(F)|$, yet neither of the error terms does. In fact, as is well known (see [S], for example), the height of $F$ is bounded below by a positive power of $|D(F)|$, so that the error term in Mahler's result actually increases as a function of $|D(F)|$.

What we are able to prove here is the following estimate for cubic forms where we not only achieve the $m^{1 / 2}$ in the error term as in (1) with an absolute constant as in (2), but this part of the error term decreases as a function of $|D(F)|$.

Theorem. Let $F(X, Y) \in \mathbb{Z}[X, Y]$ be a cubic form of discriminant $D(F)$ which is irreducible over $\mathbb{Q}$ and let $m \geq 1$. Let $N_{F}(m)$ and $A(F)$ be as above. Then

$$
\left|N_{F}(m)-m^{2 / 3} A(F)\right|<9+\frac{2008 m^{1 / 2}}{|D(F)|^{1 / 12}}+3156 m^{1 / 3} .
$$

There is nothing special about the constants 9, 2008 and 3156 appearing above; they are certainly not optimal. We have included them rather than use the $\ll$ notation to show that the constants we get are not egregiously large. Note that the main term in our theorem is smaller than the error term if $|D(F)| \gg m^{2}$, at which point the error term is $\gg \ll m^{1 / 3}$. This is not so bad since $N_{F}(m) \gg m^{1 / 3}$ for any form with the coefficient of $X^{3}$ or $Y^{3} \ll 1$. In other words, the main term in the theorem is larger than the error term exactly when one would reasonably expect it to approximate $N_{F}(m)$.

The proof of the theorem follows the typical approach of considering "small", "medium" and "large" solutions. The major improvement here on past efforts is the fairly large lower bound obtained for certain derivatives arising from the related Diophantine approximation problem. This is dealt with in the following section. Our treatment of "medium" solutions seems to be new, as well. 
Derivatives, discriminants and heights. In this section we derive relations and bounds for certain derivatives and heights arising from a cubic form $F$ in terms of the discriminant. We first fix some notation.

Let $F(X, Y) \in \mathbb{Z}[X, Y]$ be a cubic form which is irreducible over $\mathbb{Q}$. Write

$$
F(X, Y)=\prod_{i=1}^{3}\left(\delta_{i} X+\gamma_{i} Y\right)=a \prod_{i=1}^{3}\left(X-\alpha_{i} Y\right)=b \prod_{i=1}^{3}\left(Y-\beta_{i} X\right),
$$

where

$$
a=\delta_{1} \delta_{2} \delta_{3}, \quad b=\gamma_{1} \gamma_{2} \gamma_{3}, \quad \alpha_{i}=\frac{-\gamma_{i}}{\delta_{i}} \quad \text { and } \quad \beta_{i}=\alpha_{i}^{-1}
$$

Write

$$
f(X)=a \prod_{i=1}^{3}\left(X-\alpha_{i}\right)=F(X, 1) \quad \text { and } \quad g(Y)=b \prod_{i=1}^{3}\left(Y-\beta_{i}\right)=F(1, Y) .
$$

The $\delta_{i}$ 's and $\gamma_{i}$ 's are not uniquely determined by $F$, of course, but the $\alpha_{i}$ 's, $\beta_{i}$ 's, $a, b, f$ and $g$ are.

Let

$$
\Delta_{i}=\operatorname{det}\left(\begin{array}{cc}
\delta_{j} & \delta_{l} \\
\gamma_{j} & \gamma_{l}
\end{array}\right), \quad j<l, j, l \neq i
$$

and

$$
\Delta=\prod_{i=1}^{3} \Delta_{i}
$$

Then $|D(F)|=\Delta^{2} \neq 0$ (since $F$ is irreducible over $\mathbb{Q}$ ). Also, the height of $F, H(F)$, satisfies

$$
H(F)=H\left(\alpha_{i}\right)=H\left(\beta_{i}\right)=\frac{\prod_{i=1}^{3} \sqrt{\left|\delta_{i}\right|^{2}+\left|\gamma_{i}\right|^{2}}}{\operatorname{cont}(F)} .
$$

(See [S], Chap. 3, Lemma 2A.) Note that the $\left|\Delta_{i}\right|$ 's are invariant under the action of $\mathrm{GL}_{2}(\mathbb{Z})$, but $H(F)$ certainly is not.

Lemma 1. Let $F$ be a cubic form as above. After possibly applying a $T \in \mathrm{GL}_{2}(\mathbb{Z})$, we have

$$
\min _{1 \leq i \leq 3}\left\{\left|f^{\prime}\left(\alpha_{i}\right)\right|,\left|g^{\prime}\left(\beta_{i}\right)\right|\right\} \geq \frac{|D(F)|^{1 / 6}}{3 \cdot 2^{1 / 3}} .
$$

If all the $\alpha_{i}$ 's are real, then

$$
\operatorname{cont}(F) H(F) \leq 130^{1 / 2} 2^{1 / 3}|D(F)|^{1 / 3},
$$

otherwise

$$
\operatorname{cont}(F) H(F) \leq 2^{3 / 2}|D(F)|^{1 / 2} .
$$


Proof. Let $L_{i}(X, Y)=\delta_{i} X+\gamma_{i} Y$ for $i=1,2$ and 3 . We have

$$
0=\operatorname{det}\left(\begin{array}{lll}
\delta_{1} & \delta_{2} & \delta_{3} \\
\delta_{1} & \delta_{2} & \delta_{3} \\
\gamma_{1} & \gamma_{2} & \gamma_{3}
\end{array}\right)=\delta_{1} \Delta_{1}-\delta_{2} \Delta_{2}+\delta_{3} \Delta_{3},
$$

and similarly

$$
0=\gamma_{1} \Delta_{1}-\gamma_{2} \Delta_{2}+\gamma_{3} \Delta_{3}
$$

so that

$$
L_{3}(X, Y) \Delta_{3}=L_{2}(X, Y) \Delta_{2}-L_{1}(X, Y) \Delta_{1} .
$$

Also,

$$
\left|f^{\prime}\left(\alpha_{i}\right)\right|=\left|a\left(\alpha_{i}-\alpha_{j}\right)\left(\alpha_{i}-\alpha_{l}\right)\right|=\frac{|\Delta|}{\left|a\left(\alpha_{j}-\alpha_{l}\right)\right|}=\frac{|\Delta|}{\left|L_{i}(1,0) \Delta_{i}\right|}
$$

for all $i$, where $j, l \neq i$, and similarly

$$
\left|g^{\prime}\left(\beta_{i}\right)\right|=\frac{|\Delta|}{\left|L_{i}(0,1) \Delta_{i}\right|} .
$$

We first treat the case where all the $\alpha_{i}{ }^{\prime}$ 's are real. Let $P$ be the parallelogram defined by

$$
P=\left\{(x, y) \in \mathbb{R}^{2}:\left|\Delta_{i} L_{i}(x, y)\right| \leq 1 \text { for } i=1,2\right\} .
$$

Let $\lambda_{1} \leq \lambda_{2}$ be the successive minima of $P$ with respect to the integer lattice $\mathbb{Z}^{2}$. We then get a basis $\left\{\mathbf{a}_{1}, \mathbf{a}_{2}\right\}$ of $\mathbb{Z}^{2}$ that satisfies

$$
\max _{i=1,2}\left\{\left|\Delta_{i} L_{i}\left(\mathbf{a}_{1}\right)\right|\right\}=\lambda_{1}, \quad \max _{i=1,2}\left\{\left|\Delta_{i} L_{i}\left(\mathbf{a}_{2}\right)\right|\right\}=\lambda_{2} .
$$

Let $\mathbf{a}=\mathbf{a}_{1}$ and suppose without loss of generality that $\left|\Delta_{1} L_{1}(\mathbf{a})\right|=\lambda_{1}$. Let $b$ be the greatest integer part of $\Delta_{1} L_{1}\left(\mathbf{a}_{2}\right) /\left(\Delta_{1} L_{1}(\mathbf{a})\right)$ and let $\mathbf{b}=\mathbf{a}_{2}-b \mathbf{a}$. Then $\{\mathbf{a}, \mathbf{b}\}$ is a basis for $\mathbb{Z}^{2},\left|\Delta_{1} L_{1}(\mathbf{b})\right| \leq \lambda_{1}$ and $\left|\Delta_{2} L_{2}(\mathbf{b})\right| \leq 2 \lambda_{2}$. Finally, after applying a suitable $T \in \mathrm{GL}_{2}(\mathbb{Z})$ to $F$ ( $T$ is given by a and $\mathbf{b}$ ), we may assume that $\mathbf{a}=(1,0)$ and $\mathbf{b}=(0,1)$.

We conclude from this and (3) that, after applying some $T \in \mathrm{GL}_{2}(\mathbb{Z})$ and possibly reindexing, we have

$$
\begin{aligned}
& \left|\Delta_{1} L_{1}(1,0)\right|,\left|\Delta_{2} L_{2}(1,0)\right|,\left|\Delta_{1} L_{1}(0,1)\right| \leq \lambda_{1}, \\
& \left|\Delta_{3} L_{3}(1,0)\right| \leq 2 \lambda_{1}, \\
& \left|\Delta_{i} L_{i}(0,1)\right| \leq i \lambda_{2} \quad \text { for } i=2 \text { and } 3 .
\end{aligned}
$$

We need an upper bound for $\lambda_{2}$. By Minkowski's theorem, we have

$$
\lambda_{1} \lambda_{2} \leq \frac{4}{\operatorname{vol}(P)}=|\Delta| \text {. }
$$


Also, since $F$ is irreducible over $\mathbb{Q}$,

$$
|\Delta| \leq|F(1,0) \Delta|=\prod_{i=1}^{3}\left|L_{i}(1,0) \Delta_{i}\right| \leq 2 \lambda_{1}^{3}
$$

by (6). Thus

$$
\lambda_{2} \leq 2^{1 / 3}|\Delta|^{2 / 3} .
$$

The first part of Lemma 1 follows from (4)-(7). As for the height, we have by $(6)$,

$$
|\Delta| \operatorname{cont}(F) H(F) \leq \sqrt{2} \lambda_{1} \sqrt{5} \lambda_{2} \sqrt{13} \lambda_{2}=\sqrt{130}\left(\lambda_{1} \lambda_{2}\right) \lambda_{2} \leq \sqrt{130}|\Delta| \lambda_{2},
$$

so that the upper bound for the height follows from (7).

We now consider the case when the $\alpha_{i}$ 's are not all real. We may assume without loss of generality that $\delta_{3}, \gamma_{3} \in \mathbb{R}$ and that $\delta_{1}=\bar{\delta}_{2}$ and $\gamma_{1}=\bar{\gamma}_{2}$. Note that this implies $\Delta_{3} L_{3}(X, Y)=-2 \operatorname{Im}\left(\Delta_{1} L_{1}(X, Y)\right)$ by (3). We let $P$ be the parallelogram given by

$$
P=\left\{(x, y) \in \mathbb{R}^{2}:\left|\operatorname{Re}\left(\Delta_{1} L_{1}(x, y)\right)\right|,\left|\operatorname{Im}\left(\Delta_{1} L_{1}(x, y)\right)\right| \leq 1\right\}
$$

and let $\lambda_{1} \leq \lambda_{2}$ be the successive minima of $P$. We conclude similarly to above that, after applying a suitable $T \in \mathrm{GL}_{2}(\mathbb{Z})$, we have

$$
\begin{array}{ll}
\left|\Delta_{1} L_{1}(1,0)\right|=\left|\Delta_{2} L_{2}(1,0)\right| \leq \sqrt{2} \lambda_{1}, & \left|\Delta_{3} L_{3}(1,0)\right| \leq 2 \lambda_{1}, \\
\left|\Delta_{1} L_{1}(0,1)\right|=\left|\Delta_{2} L_{2}(0,1)\right| \leq \sqrt{2} \lambda_{2}, & \left|\Delta_{3} L_{3}(0,1)\right| \leq 2 \lambda_{2} .
\end{array}
$$

Similarly to above, we get $4 \lambda_{1}^{3} \geq|\Delta|$. Also,

$$
\begin{aligned}
4(\operatorname{vol}(P))^{-1} & =\left|\operatorname{det}\left(\begin{array}{ll}
\operatorname{Re}\left(\Delta_{1} \delta_{1}\right) & \operatorname{Im}\left(\Delta_{1} \delta_{1}\right) \\
\operatorname{Re}\left(\Delta_{1} \gamma_{1}\right) & \operatorname{Im}\left(\Delta_{1} \gamma_{1}\right)
\end{array}\right)\right|=\left|\operatorname{Im}\left(\Delta_{1} \delta_{1} \overline{\Delta_{1} \gamma_{1}}\right)\right| \\
& =\frac{1}{2}\left|\operatorname{det}\left(\begin{array}{ll}
\Delta_{1} \delta_{1} & \overline{\Delta_{1} \delta_{1}} \\
\Delta_{1} \gamma_{1} & \overline{\Delta_{1} \gamma_{1}}
\end{array}\right)\right|=\frac{1}{2}\left|\operatorname{det}\left(\begin{array}{cc}
\Delta_{1} \delta_{1} & \Delta_{2} \delta_{2} \\
\Delta_{1} \gamma_{1} & \Delta_{2} \gamma_{2}
\end{array}\right)\right|=\frac{|\Delta|}{2} .
\end{aligned}
$$

Thus,

$$
\lambda_{2} \leq \frac{4}{\lambda_{1} \operatorname{vol}(P)} \leq \frac{|\Delta|^{2 / 3}}{2^{1 / 3}}
$$

The remainder of the lemma follows from this and (8).

Approximations and a gap argument. In this section we prove two auxiliary results. They are routine Diophantine approximation fare, but we have included them for completeness. We continue with the notation established above.

Lemma 2. Let $F$ be a cubic form as in the Theorem and suppose $(x, y) \in$ $\mathbb{R}^{2}$ satisfies $|F(x, y)| \leq m$. If $y \neq 0$, then 


$$
\left|\alpha_{i}-\frac{x}{y}\right| \leq \frac{4 m}{\left|y^{3} f^{\prime}\left(\alpha_{i}\right)\right|}
$$

for some $1 \leq i \leq 3$. Similarly, if $x \neq 0$, then for some $i$,

$$
\left|\beta_{i}-\frac{y}{x}\right| \leq \frac{4 m}{\left|x^{3} g^{\prime}\left(\beta_{i}\right)\right|}
$$

Pro of. Suppose $y \neq 0$ and choose $i$ so that $\left|\alpha_{i}-(x / y)\right|$ is smallest. For $j \neq i$ we have

$$
2\left|\alpha_{j}-\frac{x}{y}\right| \geq\left|\alpha_{j}-\frac{x}{y}\right|+\left|\alpha_{i}-\frac{x}{y}\right| \geq\left|\alpha_{j}-\alpha_{i}\right|
$$

Thus,

$$
\frac{m}{\left|y^{3}\right|} \geq\left|\frac{F(x, y)}{y^{3}}\right|=|f(x / y)|=|a| \prod_{j=1}^{3}\left|\alpha_{j}-\frac{x}{y}\right| \geq\left|\alpha_{i}-\frac{x}{y}\right| \frac{\left|f^{\prime}\left(\alpha_{i}\right)\right|}{4}
$$

by (4). The proof for $x \neq 0$ is the same with the obvious changes.

From now on, when we write $x / y \in \mathbb{Q}$ it is assumed that $x$ and $y$ are relatively prime integers with $y>0$.

Lemma 3. Let $\alpha \in \mathbb{C}$ and let $A, B, C \in \mathbb{R}$ with $C \geq B>A>0$. The number of $x / y \in \mathbb{Q}$ with $|\alpha-(x / y)| \leq A /\left(2 y^{3}\right)$ and $B \leq y \leq C$ is no greater than

$$
1+\log _{2}\left(\frac{\log C-\log A}{\log B-\log A}\right) .
$$

P r o of. Let $x_{0} / y_{0}, x_{1} / y_{1}, \ldots$ be the distinct rational numbers satisfying the hypotheses of the lemma, arranged so that $y_{0} \leq y_{1} \leq \ldots$ We claim that $y_{n} \geq A(B / A)^{2^{n}}$. This is trivially true for $n=0$. We proceed by induction on $n$. We have

$$
\begin{aligned}
\frac{1}{y_{n} y_{n+1}} & \leq\left|\frac{x_{n}}{y_{n}}-\frac{x_{n+1}}{y_{n+1}}\right| \leq\left|\frac{x_{n}}{y_{n}}-\alpha\right|+\left|\frac{x_{n+1}}{y_{n+1}}-\alpha\right| \\
& \leq \frac{A}{2 y_{n}^{3}}+\frac{A}{2 y_{n+1}^{3}} \leq \frac{A}{y_{n}^{3}},
\end{aligned}
$$

so that $y_{n+1} \geq A^{-1} y_{n}^{2}$. The claim follows from this and the induction hypothesis.

By the claim, if $y_{n} \leq C$, then $C \geq A(B / A)^{2^{n}}$. This implies that

$$
\log _{2}\left(\frac{\log C-\log A}{\log B-\log A}\right) \geq n,
$$

proving the lemma.

Small solutions. Since $A(F),|D(F)|$ and $N_{F}(m)$ are all invariant under the action of $\mathrm{GL}_{2}(\mathbb{Z})$, we may assume without loss of generality that 
our form satisfies the conclusions of Lemma 1. Specifically, we assume from now on that

$$
\left|f^{\prime}\left(\alpha_{i}\right)\right|,\left|g^{\prime}\left(\beta_{i}\right)\right| \geq \frac{|D(F)|^{1 / 6}}{2^{1 / 3} 3}=c_{1}|D(F)|^{1 / 6}
$$

and that

$$
H\left(\alpha_{i}\right)=H\left(\beta_{i}\right)=H(F) \leq 130^{1 / 2} 2^{1 / 3}|D(F)|^{1 / 2}=c_{2}|D(F)|^{1 / 2}
$$

for $i=1,2$ and 3 .

Lemma 4. Let $N_{F}^{\prime}(m)$ denote the number of solutions to $|F(x, y)| \leq m$ with $\max \{|x|,|y|\} \leq m^{1 / 2} /|D(F)|^{1 / 12}$. Then

$$
\left|N_{F}^{\prime}(m)-m^{2 / 3} A(F)\right|<9+\frac{375 m^{1 / 2}}{|D(F)|^{1 / 12}} .
$$

Proof. For the time being, let $M=m^{1 / 2} /|D(F)|^{1 / 12}$. By [D] we have

$$
\left|N_{F}^{\prime}(m)-B_{F}(m)\right| \leq 9+12 M,
$$

where $B_{F}(m)$ denotes the area of the region

$$
\left\{(x, y) \in \mathbb{R}^{2}:|F(x, y)| \leq m, \max \{|x|,|y|\} \leq M\right\} .
$$

Let $B_{F}^{\prime}(m)$ denote the area of the region

$$
\left\{(x, y) \in \mathbb{R}^{2}:|F(x, y)| \leq m, \max \{|x|,|y|\}>M\right\},
$$

so that $B_{F}(m)+B_{F}^{\prime}(m)=m^{2 / 3} A(F)$.

By $(9)$,

$$
\begin{aligned}
\int_{|y|>M} \int_{\left|x-\alpha_{i} y\right|<4 m /\left(y^{2}\left|f^{\prime}\left(\alpha_{i}\right)\right|\right)} d x d y & =\frac{8 m}{\left|f^{\prime}\left(\alpha_{i}\right)\right|} \int_{|y|>M} \frac{1}{y^{2}} d y \\
& =\frac{16 m}{M\left|f^{\prime}\left(\alpha_{i}\right)\right|} \leq \frac{16 m^{1 / 2}}{c_{1}|D(F)|^{1 / 12}}
\end{aligned}
$$

and similarly

$$
\int_{|x|>M} \int_{\left|y-\beta_{i} x\right|<4 m /\left(x^{2}\left|g^{\prime}\left(\beta_{i}\right)\right|\right)} d y d x \leq \frac{16 m^{1 / 2}}{c_{1}|D(F)|^{1 / 12}}
$$

for any $i$. Thus, by Lemma 2 ,

$$
B_{F}^{\prime}(m) \leq \frac{6 \cdot 16 m^{1 / 2}}{c_{1}|D(F)|^{1 / 12}}<\frac{363 m^{1 / 2}}{|D(F)|^{1 / 12}} .
$$

In conclusion, we have

$$
\begin{aligned}
\left|N_{F}^{\prime}(m)-m^{2 / 3} A_{F}(m)\right| & \leq\left|N_{F}^{\prime}(m)-B_{F}(m)\right|+\left|B_{F}(m)-m^{2 / 3} A_{F}(m)\right| \\
& \leq 9+\frac{12 m^{1 / 2}}{|D(F)|^{1 / 12}}+B_{F}^{\prime}(m)<9+\frac{375 m^{1 / 2}}{|D(F)|^{1 / 12}} .
\end{aligned}
$$


Medium solutions. For the time being, fix an $\alpha$ amongst $\alpha_{1}, \alpha_{2}$ and $\alpha_{3}$. We will say an $(x, y) \in \mathbb{Z}^{2}$ with $y \neq 0$ is closest to $\alpha$ if $|\alpha-(x / y)|$ is minimal, i.e., no greater than $\left|\alpha_{i}-(x / y)\right|$ for any $i$. While this does not rule out $(x, y)$ being closest to two $\alpha_{i}$ 's, it is closest to at least one of them.

Lemma 5. Let $B>0$ and let $\left(x_{0}, y_{0}\right)$ be a solution to $|F(x, y)| \leq m$ closest to $\alpha$ with $B \leq y_{0}<2 B$. The number of solutions $(x, y) \in \mathbb{Z}^{2}$ to $|F(x, y)| \leq m$ closest to $\alpha$ with $B \leq y<2 B$ and $(x, y)$ not a scalar multiple of $\left(x_{0}, y_{0}\right)$ is less than $18 m /\left(B c_{1}|D(F)|^{1 / 6}\right)$. The number of such solutions that are a scalar multiple of $\left(x_{0}, y_{0}\right)$ is no greater than $g=\operatorname{gcd}\left(x_{0}, y_{0}\right)$.

Proof. Write $g\left(x^{\prime}, y^{\prime}\right)=\left(x_{0}, y_{0}\right)$, where $g$ is the greatest common divisor of $x_{0}$ and $y_{0}$. Note that $\left|F\left(x^{\prime}, y^{\prime}\right)\right| \leq m g^{-3}$ and $\left(x^{\prime}, y^{\prime}\right)$ is closest to $\alpha$. Let $a^{\prime}, b^{\prime} \in \mathbb{Z}$ satisfy $a^{\prime} y^{\prime}-b^{\prime} x^{\prime}=1$.

Given any solution $(x, y)$ satisfying the hypotheses, we get a unique pair $a, b \in \mathbb{Z}$ with

$$
x=a a^{\prime}+b x^{\prime}, \quad y=a b^{\prime}+b y^{\prime}, \quad \text { and } \quad a=y^{\prime} x-x^{\prime} y .
$$

Lemma 2 gives

$$
\begin{aligned}
|g a| & =\left|x_{0} y-y_{0} x\right|=y y_{0}\left|\frac{x_{0}}{y_{0}}-\frac{x}{y}\right| \\
& \leq y y_{0}\left(\frac{4 m}{y_{0}^{3}\left|f^{\prime}(\alpha)\right|}+\frac{4 m}{y^{3}\left|f^{\prime}(\alpha)\right|}\right)<\frac{9 m}{B\left|f^{\prime}(\alpha)\right|} .
\end{aligned}
$$

Further, for a given $a$ we have

$$
\frac{B-a b^{\prime}}{y^{\prime}} \leq \frac{y-a b^{\prime}}{y^{\prime}}=b<\frac{2 B-a b^{\prime}}{y^{\prime}},
$$

giving no more than $B / y^{\prime}=g B / y_{0} \leq g$ possible values of $b$. Note that $(x, y)$ is a scalar multiple of $\left(x_{0}, y_{0}\right)$ if and only if $a=0$. Lemma 5 now follows from (9).

LEMMA 6 . The number of solutions $(x, y)$ to $|F(x, y)| \leq m$ closest to $\alpha$ with $m^{1 / 2}|D(F)|^{1 / 12} \leq y<16 m /\left(c_{1}|D(F)|^{1 / 6}\right)$ is no greater than

$$
\frac{36 m^{1 / 2}}{c_{1}|D(F)|^{1 / 12}}+144 m^{1 / 3} \text {. }
$$

Proof. Let $B_{0}=m^{1 / 2} /|D(F)|^{1 / 12}$ and let $N$ be least such that $2^{N} B_{0} \geq$ $16 m /\left(c_{1}|D(F)|^{1 / 6}\right)$. We will use Lemma 5 , counting those solutions with $2^{i} B_{0} \leq y<2^{i+1} B_{0}$ for $i=0, \ldots, N-1$. For some, possibly not all, $i=0, \ldots, N-1$, we have positive integers $g_{i}$ and $x_{i} / y_{i} \in \mathbb{Q}$ with $2^{i} B_{0} \leq$ $g_{i} y_{i}<2^{i+1} B_{0},\left|F\left(x_{i}, y_{i}\right)\right| \leq m g_{i}^{-3}$ and $\left(x_{i}, y_{i}\right)$ closest to $\alpha$. Setting $g_{i}=0$ 
for all other $i$, we get less than

$\sum_{i=0}^{N-1} \frac{18 m}{2^{i} B_{0} c_{1}|D(F)|^{1 / 6}}+g_{i}<\frac{36 m}{B_{0} c_{1}|D(F)|^{1 / 6}}+\sum_{i=0}^{N-1} g_{i}=\frac{36 m^{1 / 2}}{c_{1}|D(F)|^{1 / 12}}+\sum_{i=0}^{N-1} g_{i}$

possible solutions.

Now $2^{N} B_{0}<32 B_{0}^{2} / c_{1}$, so that

$$
N<5-\log _{2} c_{1}+\log _{2} B_{0}<5-\log _{2} c_{1}+(21 /(2 \log 2)) m^{1 / 21} .
$$

Thus,

$$
\begin{aligned}
\sum_{g_{i} \leq 4 c_{1}^{-1 / 3} m^{2 / 7}} g_{i} & <\left(5-\log _{2} c_{1}+(21 /(2 \log 2)) m^{1 / 21}\right) 4 c_{1}^{-1 / 3} m^{2 / 7} \\
& \leq\left(5-\log _{2} c_{1}+(21 /(2 \log 2))\right) 4 c_{1}^{-1 / 3} m^{1 / 3}<138 m^{1 / 3} .
\end{aligned}
$$

It remains to estimate the sum over those $g_{i}$ greater than $4 c_{1}^{-1 / 3} m^{2 / 7}$. If $g_{i}>4 c_{1}^{-1 / 3} m^{2 / 7}$, then we have

$$
\left|\alpha-\frac{x_{i}}{y_{i}}\right| \leq \frac{4 m}{\left(g_{i} y_{i}\right)^{3} c_{1}|D(F)|^{1 / 6}}<\frac{m^{1 / 7}}{16 y_{i}^{3}|D(F)|^{1 / 6}} .
$$

Also, since $g_{i}^{-3} m \geq\left|F\left(x_{i}, y_{i}\right)\right| \geq 1$, we have $g_{i} \leq m^{1 / 3}$ and $y_{i} \geq B_{0} m^{-1 / 3}=$ $m^{1 / 6} /|D(F)|^{1 / 12}$. We now use Lemma 3 , with $A=m^{1 / 7} /\left(8|D(F)|^{1 / 6}\right), B=$ $m^{1 / 6} /|D(F)|^{1 / 12}$ and $C=16 m /\left(c_{1}|D(F)|^{1 / 6}\right)$. According to Lemma 3, we have no greater than

$$
\begin{aligned}
1+\log _{2}\left(\frac{\log _{2} C-\log _{2} A}{\log _{2} B-\log _{2} A}\right) & \leq 1+\log _{2}\left(\frac{7+(6 / 7) \log _{2} m-\log _{2} c_{1}}{3+(1 / 42) \log _{2} m}\right) \\
& <1+\log _{2}\left((7 / 3)+36-(1 / 3) \log _{2} c_{1}\right)<7
\end{aligned}
$$

possible $x_{i} / y_{i}$ with $g_{i}>4 c_{1}^{-1 / 3} m^{2 / 7}$. Thus,

$$
\sum_{g_{i}>4 c_{1}^{-1 / 3} m^{2 / 7}} g_{i} \leq 6 m^{1 / 3} .
$$

All told, we have less than

$$
\frac{36 m^{1 / 2}}{c_{1}|D(F)|^{1 / 12}}+\sum_{i=0}^{N-1} g_{i}<\frac{36 m^{1 / 2}}{c_{1}|D(F)|^{1 / 12}}+144 m^{1 / 3}
$$

possible solutions.

Lemma 7. Let $N_{F}^{\prime \prime}(m)$ denote the number of solutions $(x, y) \in \mathbb{Z}^{2}$ to $|F(x, y)| \leq m$ with

$$
\frac{m^{1 / 2}}{|D(F)|^{1 / 12}}<\max \{|x|,|y|\}<\frac{16 m}{c_{1}|D(F)|^{1 / 6}} .
$$


Then

$$
N_{F}^{\prime \prime}(m)<12\left(\frac{36 m^{1 / 2}}{c_{1}|D(F)|^{1 / 12}}+144 m^{1 / 3}\right)<\frac{1633 m^{1 / 2}}{|D(F)|^{1 / 12}}+1728 m^{1 / 3} .
$$

Proof. Multiplying by two the estimate in Lemma 6 above counts all those solutions with

$$
m^{1 / 2}|D(F)|^{1 / 12} \leq|y|<16 m /\left(c_{1}|D(F)|^{1 / 6}\right)
$$

that are closest to $\alpha$. Multiplying by 3 takes care of the different possibilities for $\alpha$. Finally, the arguments above are entirely symmetric with respect to $x$ and $y$, i.e., the same estimates hold for counting solutions $(x, y)$ with $x \neq 0$ and $y / x$ closest to $\beta=\beta_{i}$ for some $i$.

\section{Large solutions}

LEMMA 8. Fix an $i$ between 1 and 3 and suppose $(x, y) \in \mathbb{Z}^{2}$ is a solution to $|F(x, y)| \leq m$ closest to $\alpha_{i}$ with $y \geq 16 m /\left(c_{1}|D(F)|^{1 / 6}\right)$. Then there is an $x^{\prime} / y^{\prime} \in \mathbb{Q}$ with $(x, y)=g\left(x^{\prime}, y^{\prime}\right)$, where $g=\operatorname{gcd}(x, y)$ and $\left(x^{\prime}, y^{\prime}\right)$ is closest to $\alpha_{i}$. Further,

$$
\left|\alpha_{i}-\frac{x^{\prime}}{y^{\prime}}\right| \leq \frac{4 m}{\left(g y^{\prime}\right)^{3} c_{1}|D(F)|^{1 / 6}}, \quad y^{\prime} \geq \frac{16 m}{c_{1} g|D(F)|^{1 / 6}}
$$

and $g \leq m^{1 / 3}$.

P r o of. The first statement is clear given our conventions mentioned above about writing elements of $\mathbb{Q}$. Certainly $x^{\prime} / y^{\prime}$ is closest to $\alpha_{i}$, so that the second statement follows from Lemma 1 and (9). Finally, we have $g^{3} \leq g^{3}\left|F\left(x^{\prime}, y^{\prime}\right)\right|=|F(x, y)| \leq m$.

For the next two lemmas, fix an $\alpha$ as in the last section and a positive integer $g \leq m^{1 / 3}$.

LEMMA 9. Let

$$
C=\max \left\{\left(8 m /\left(g^{3} c_{1}|D(F)|^{1 / 6}\right)\right)^{4},\left(8^{3} H(\alpha)\right)^{2 \cdot 54^{3}}\right\} .
$$

The number of $x / y \in \mathbb{Q}$ with $16 m /\left(c_{1} g|D(F)|^{1 / 6}\right) \leq y \leq C$ satisfying

$$
\left|\alpha-\frac{x}{y}\right| \leq \frac{4 m}{y^{3} g^{3} c_{1}|D(F)|^{1 / 6}}
$$

is less than

$$
25.6+\log _{2}\left(4-\log _{2} c_{1}+\frac{\log _{2} m}{1+2 \log _{2} g}\right) .
$$

Proof. Let

$$
A=\frac{8 m}{g^{3} c_{1}|D(F)|^{1 / 6}} \quad \text { and } \quad B=\max \left\{2 g^{2} A, 1\right\} .
$$


Note that by (10),

$$
C \leq \max \left\{A^{4},\left(c_{2}^{2} 8^{6}|D(F)|\right)^{54^{3}}\right\} .
$$

We are then counting the number of $x / y \in \mathbb{Q}$ with $B \leq y \leq C$ satisfying $|\alpha-(x / y)| \leq A /\left(2 y^{3}\right)$. We estimate the number of such $x / y$ using Lemma 3 .

First suppose $B>1$, so that $\log _{2} B-\log _{2} A=1+2 \log _{2} g$ and $16 m>$ $c_{1} g|D(F)|^{1 / 6}$. If $C=A^{4}$, then

$$
\frac{\log _{2} C-\log _{2} A}{\log _{2} B-\log _{2} A}=\frac{3 \log _{2} A}{1+2 \log _{2} g} \leq 9-3 \log _{2} c_{1}+\frac{3 \log _{2} m}{1+2 \log _{2} g},
$$

since $A \leq 8 m / c_{1}$. If $C>A^{4}$, then by (11),

$$
\begin{aligned}
\log _{2} C & \leq 54^{3}\left(2 \log _{2} c_{2}+18+\log _{2}|D(F)|\right) \\
& \leq 54^{3}\left(2 \log _{2} c_{2}+18+6\left(4-\log _{2} c_{1}+\log _{2} m\right)\right) .
\end{aligned}
$$

Thus,

$$
\begin{aligned}
\frac{\log _{2} C-\log _{2} A}{\log _{2} B-\log _{2} A} & \leq 1+\frac{\log _{2} C}{1+2 \log _{2} g} \\
& \leq 54^{3}\left(2 \log _{2} c_{2}-6 \log _{2} c_{1}+43+\frac{6 \log _{2} m}{1+2 \log _{2} g}\right) .
\end{aligned}
$$

Now suppose $B=1$. Then $A \leq 1 /\left(2 g^{2}\right)$ and

$$
\log _{2} C \leq 54^{3}\left(2 \log _{2} c_{2}+18+\log _{2}|D(F)|\right)
$$

by (11). We also have $|D(F)| \geq 16$, since $|D(F)| \in \mathbb{Z}$ and

$$
1 \geq 16 m /\left(g c_{1}|D(F)|^{1 / 6}\right) \geq 16 /|D(F)| \text {. }
$$

We get

$$
\frac{\log _{2} C}{\log _{2}|D(F)|}<54^{3}\left(2 \log _{2} c_{2}+19\right)
$$

and

$$
\begin{aligned}
\frac{\log _{2}|D(F)|}{-6 \log _{2} A} & =\frac{\log _{2}\left(8 m /\left(c_{1} g^{3}\right)\right)-\log _{2} A}{-\log _{2} A} \leq 1+\frac{3+\log _{2} m-\log _{2} c_{1}}{-\log _{2} A} \\
& \leq 4-\log _{2} c_{1}+\frac{\log _{2} m}{1+2 \log _{2} g} .
\end{aligned}
$$

Thus,

$$
\begin{aligned}
\frac{\log _{2} C-\log _{2} A}{\log _{2} B-\log _{2} A} & =1+\frac{\log _{2} C}{-\log _{2} A} \\
& <6 \cdot 54^{3}\left(2 \log _{2} c_{2}+19\right)\left(4-\log _{2} c_{1}+\frac{\log _{2} m}{1+2 \log _{2} g}\right) .
\end{aligned}
$$


In all cases we have

$$
\frac{\log _{2} C-\log _{2} A}{\log _{2} B-\log _{2} A}<6 \cdot 54^{3}\left(2 \log _{2} c_{2}+19\right)\left(4-\log _{2} c_{1}+\frac{\log _{2} m}{1+2 \log _{2} g}\right),
$$

so that

$$
1+\log _{2}\left(\frac{\log _{2} C-\log _{2} A}{\log _{2} B-\log _{2} A}\right)<25.6+\log _{2}\left(4-\log _{2} c_{1}+\frac{\log _{2} m}{1+2 \log _{2} g}\right) .
$$

Lemma 9 follows from this and Lemma 3.

Lemma 10. Let $C$ be as in Lemma 9. The number of $x / y \in \mathbb{Q}$ with $y>C$ and $y \geq|x|$ satisfying

$$
\left|\alpha-\frac{x}{y}\right| \leq \frac{4 m}{y^{3} g^{3} c_{1}|D(F)|^{1 / 6}}
$$

is no greater than 25 .

Proof. For $x / y \in \mathbb{Q}$, set $H(x / y)=\sqrt{x^{2}+y^{2}}$ (recall our conventions about $x / y \in \mathbb{Q}$ ). In particular, for the $x / y \in \mathbb{Q}$ considered in Lemma 10, $y \leq H(x, y)<2 y$.

By Theorem $6 \mathrm{~A}$ of $[\mathrm{S}]$, Chapter 2 (using $m=2$ and $\chi=1 / 9$ ), there is some $B>0$ such that all $x / y \in \mathbb{Q}$ satisfying

$$
\left|\alpha-\frac{x}{y}\right|<\frac{1}{H(x / y)^{10 \sqrt{6} / 9}}
$$

have either $H(x / y)<\left(8^{3} H(\alpha)\right)^{2 \cdot 54^{3}}$ or $B \leq H(x / y)<B^{4 \cdot 54^{3}}$. Note that all $x / y \in \mathbb{Q}$ satisfying the hypotheses of Lemma 10 have

$$
\begin{aligned}
\left|\alpha-\frac{x}{y}\right| & \leq \frac{4 m}{(y g)^{3} c_{1}|D(F)|^{1 / 6}}<\frac{4 m}{y^{11 / 4} C^{1 / 4} g^{3} c_{1}|D(F)|^{1 / 6}} \\
& \leq \frac{1}{2 y^{11 / 4}}<\frac{1}{H(x / y)^{10 \sqrt{6} / 9}}
\end{aligned}
$$

and $H(x / y)>\left(8^{3} H(\alpha)\right)^{2 \cdot 54^{3}}$.

A standard gap argument (Lemma $8 \mathrm{~B}$ of $[\mathrm{S}]$, Chapter 2, for example) shows that the number of $x / y \in \mathbb{Q}$ with

$$
\left|\alpha-\frac{x}{y}\right|<\frac{1}{2 y^{11 / 4}}
$$

and $B \leq y<B^{4 \cdot 54^{3}}$ is no greater than

$$
1+\frac{\log \left(\log \left(B^{4 \cdot 54^{3}}\right) / \log B\right)}{\log (7 / 4)}<25
$$

and the number with $B / 2 \leq y<B$ is at most 1 . This proves Lemma 10 . 
Lemma 11. Let $N_{F}^{\prime \prime \prime}(m)$ denote the number of solutions $(x, y) \in \mathbb{Z}^{2}$ to $|F(x, y)| \leq m$ with

$$
\max \{|x|,|y|\} \geq \frac{16 m}{c_{1}|D(F)|^{1 / 6}} .
$$

Then $N_{F}^{\prime \prime \prime}(m)<1428 m^{1 / 3}$.

Proof. Fix an $i$ between 1 and 3. By Lemmas 8-10, the number of solutions closest to $\alpha_{i}$ with

$$
\max \{|x|,|y|\}=y \geq \frac{16 m}{c_{1}|D(F)|^{1 / 6}}
$$

is less than

$$
\sum_{g \leq m^{1 / 3}} 50.6+\log _{2}\left(4-\log _{2} c_{1}+\frac{\log _{2} m}{1+2 \log _{2} g}\right) .
$$

Now

$$
\begin{aligned}
\sum_{g \leq m^{1 / 6}} 50.6+\log _{2}(4- & \left.\log _{2} c_{1}+\frac{\log _{2} m}{1+2 \log _{2} g}\right) \\
& \leq m^{1 / 6}\left(50.6+\log _{2}\left(4-\log _{2} c_{1}+\log _{2} m\right)\right) \\
& <m^{1 / 6}\left(50.6+\log _{2}\left(8-2 \log _{2} c_{1}\right)+\log _{2} m\right) \\
& <m^{1 / 6}\left(54.4+8.7 m^{1 / 6}\right)<64 m^{1 / 3}
\end{aligned}
$$

and

$$
\begin{aligned}
& \sum_{m^{1 / 6}<g \leq m^{1 / 3}} 50.6+\log _{2}\left(4-\log _{2} c_{1}+\frac{\log _{2} m}{1+2 \log _{2} g}\right) \\
&<m^{1 / 3}\left(50.6+\log _{2}\left(4-\log _{2} c_{1}+3\right)\right)<55 m^{1 / 3} .
\end{aligned}
$$

Thus, the number of solutions closest to $\alpha_{i}$ with

$$
\max \{|x|,|y|\}=y>\frac{16 m}{c_{1}|D(F)|^{1 / 6}}
$$

is less than $119 m^{1 / 3}$. Moreover, the exact same estimates hold by the symmetry of our argument for the number of solutions closest to $\beta_{i}$ with

$$
\max \{|x|,|y|\}=x>\frac{16 m}{c_{1}|D(F)|^{1 / 6}} .
$$

Arguing exactly as in the proof of Lemma 7, we get

$$
N_{F}^{\prime \prime \prime}(m)<12 \cdot 119 m^{1 / 3}=1428 m^{1 / 3} .
$$

Proof of Theorem. By Lemmas 7 and 11 we have

$$
N_{F}(m)-N_{F}^{\prime}(m)=N_{F}^{\prime \prime}(m)+N_{F}^{\prime \prime \prime}(m)<\frac{1633 m^{1 / 2}}{|D(F)|^{1 / 12}}+3156 m^{1 / 3} .
$$


Also,

$$
\begin{aligned}
\left|N_{F}(m)-m^{2 / 3} A(F)\right| & \leq\left|N_{F}(m)-N_{F}^{\prime}(m)\right|+\left|N_{F}^{\prime}(m)-m^{2 / 3} A(F)\right| \\
& \leq\left|N_{F}(m)-N_{F}^{\prime}(m)\right|+9+\frac{375 m^{1 / 2}}{|D(F)|^{1 / 12}}
\end{aligned}
$$

by Lemma 4. The Theorem follows.

\section{References}

[B1] M. Bean, Bounds for the number of solutions of the Thue equation, M. thesis, Univ. of Waterloo, 1988.

[B2] -, An isoparametric inequality for the area of plane regions defined by binary forms, Compositio Math. 92 (1994), 115-131.

[D] H. Davenport, On a principle of Lipschitz, J. London Math. Soc. 26 (1951), 179-183.

[M] K. Mahler, Zur Approximation algebraischer Zahlen III, Acta Math. 62 (1934), 91-166.

[MS1] J. Mueller and W. M. Schmidt, Trinomial Thue equations and inequalities, J. Reine Angew. Math. 379 (1987), 76-99.

[MS2] -, - The generalized Thue inequality, Compositio Math. 96 (1995), 331-344.

[S] W. Schmidt, Diophantine Approximation, Lecture Notes in Math. 1467, Springer, New York, 1991.

[T1] J. L. Thunder, The number of solutions to cubic Thue inequalities, Acta Arith. 66 (1994), 237-243.

[T2] -, On Thue inequalities and a conjecture of Schmidt, J. Number Theory 52 (1995), 319-328.

Mathematical Sciences Department

Northern Illinois University

DeKalb, Illinois 60115

U.S.A.

E-mail: jthunder@math.niu.edu 